\title{
RANGOS REFERENCIALES DEL ÍNDICE DE PULSATILIDAD DE LA ARTERIA UTERINA EN FETOS SANOS INSTITUTO NACIONAL MATERNO PERINATAL 2010 A 2016
}

\author{
Oscar Antonio Limay Rios ${ }^{1}$, Karin Jessica Laucatta Alarcon ${ }^{2}$, Jaime Ingar Pinedo ${ }^{3}$,
} Erasmo Huertas Tachinno ${ }^{4}$, Walter Castillo Urquiaga ${ }^{4}$, Walter Ventura Laveriano ${ }^{4}$, Mario Zarate Girao ${ }^{4}$

\begin{abstract}
RESUMEN
Objetivo: Describir los valores de referencia índice pulsatilidad de la arteria uterina entre las semanas 11 - 41 de gestación de fetos sanos en Instituto Nacional materno perinatal en el período 2010 a 2016. Diseño: Estudio cuantitativo, observacional, descriptivo, transversal y retrospectivo. Participantes: 7020 fetos sanos de madres de bajo riesgo con evaluación doppler entre 11 a 41 semanas de gestación. Principal medida de resultado: Rangos de referencia del índice de pulsatilidad de la arteria uterina. Resultados: Los índices de pulsatilidad medio (P50) de nuestro estudio fueron menores en relación con los estudios realizados por Krampl, donde la diferencia es evidente en las primeras semanas como al final de la gestación, coincidiendo con nuestros índices a las 24 y 33, 34 semanas. El presente estudio tuvo una diferencia significativa con la P50 de Gómez y Figueras entre 12 a 24 semanas, así como interacción de 27 a 41 semanas. Conclusiones: Los rangos de referencia para el percentil 5, 50 y 95 del índice de pulsatilidad de la arteria uterina se establecieron en fetos sanos, demostrando que tienen un valor clínico en la detección de fetos en buenas condiciones si el flujo doppler se encuentra dentro de parámetros normales. Lo cual permite hacer un seguimiento especial a los casos que se encuentre fuera de estos rangos por el riesgo que existe de presentar restricción del crecimiento intrauterino o macrosomía fetal. Las curvas doppler muestran una disminución progresiva hasta las últimas etapas del embarazo. Los índices de pulsatilidad de las arterias uterinas medidas entre las semanas 11 y 41 de este estudio se encuentran más bajas que los valores reportadas en el estudio Krampl y en el estudio de Gómez y Figueras.
\end{abstract}

Palabras clave: Rangos de referencia; Ïndice de pulsatilidad; Curva normal del índice de pulsatilidad; Feto sano (Fuente DeCS BIREME).

\section{REFERENTIAL RANGE OF THE PULSATILITY INDEX OF THE UTERINE ARTERY IN FETUS HEALTHY MATERNAL PERINATAL NATIONAL INSTITUTE 2010 TO 2016}

\begin{abstract}
Objectives: Set the values for reference index pulsatilidad of artery uterine between 11-41 weeks of gestation in fetuses healthy in the maternal perinatal national institute in the period 2010 to 2016. Desing: Retrospective Cross-sectional descriptive study. Participants: Healthy fetuses. Main outcome measures: Reference ranges of the uterine artery pulsatility index. Results: The mean pulsatility indexes (P50) of our study were lower in relation to the studies performed by Krampl, where the difference is evident in the first weeks tan at the end of gestation, coinciding whith our indeces at 24 and 33,34 weeks. The present study had a significant difference with the P50 of Gomez and Figueras between 12 to 24 weeks, as well as interaction from 27 to 41 weeks. Conclusions: The reference ranges for the 5th, 50th and 95th percentile of the uterine artery pulsatility index were established in healthy fetuses, showing that they have a clinical value in the detection of fetuses in good condition. The doppler curves show a progressive decrease until the last stages of pregnancy. Pulsatility rates of uterine arteries measured between weeks 11 and 41 of this study are found to be lower than the rates reported in the Krampl study and the Gómez and Figueras study.
\end{abstract}

Key words: Reference ranges; Pulsatility index; Normal curve of the pulsatility index; Healthy fetus (Source: MeSH NLM).

\section{INTRODUCCIÓN}

El incremento en los últimos años de recién nacidos con restricción de crecimiento intrauterino y/o macrosomía fetal y el mayor riesgo de complicaciones perinatales que esto conlleva, obliga a realizar evaluaciones para detectar la condición fetal durante todo el embarazo, dato importante para poder anticiparnos a problemas que el neonato pueda presentar en forma precoz ${ }^{1}$.

En los países en vías de desarrollo la restricción del crecimiento intrauterino es el mayor contribuyente de bajo peso con un $11 \%^{2}$ y la incidencia de macrosomía, según lo informado por algunos autores, oscila entre el 4,7 y $16,4 \%^{3}$.
En el Perú la incidencia de restricción del crecimiento intrauterino es de $10.14 \% \%^{2,4}$. A nivel hospitalario la incidencia es variable, 7,6\% en Lima metropolitana, 9,4\% en la costa, $11,8 \%$ en selva y $14,6 \%$ en la sierra ${ }^{2}$. En el INMP varia de 4 a $15 \%$; La tasa de macrosomía en nuestro país tiene una expectativa de crecimiento en los últimos años, con una prevalencia de 14,34\% en Lima ${ }^{6}$. La incidencia nacional hospitalaria de macrosomía fetal es de $5,7 \%$, siendo aún más elevada en la costa ${ }^{3}$. Según la base de datos del Instituto Nacional Materno Perinatal, ha pasado de un $6 \%$ en el año de 1995 a casi un $9 \%$ para el 2015. Es decir, se aprecia claramente que la frecuencia de niños macrosómicos se ha incrementado ${ }^{6}$, representando una pérdida perinatal de alrededor del $7,2 \%$.

\footnotetext{
Médico Gineco-Obstetra Asistente del Servicio de Medicina Fetal. Jefe de Unidad de Investigación del Instituto Nacional Materno Perinatal. Lima-Perú

Estudiante de Facultad de Medicina Humana de la Universidad Privada San Juan Bautista.Lima-Perú.

Médico Gineco-Obstetra Jefe del Servicio de Medicina Fetal del Instituto Nacional Materno Perinatal. Lima-Perú.

4 Médico Gineco-Obstetra Asistente del Servicio de Medicina Fetal del Instituto Nacional Materno Perinatal. Lima-Perú
}

Recibido: 01-12-16 Aprobado: 30-06-17 
El crecimiento intrauterino que el feto presente de acuerdo a su edad gestacional es importante porque los niños que no logren un crecimiento adecuado por lo general, presentarán en un futuro múltiples problemas, tanto en el periodo neonatal como en la niñez, la adolescencia y aun en la edad adulta. La estimación del desarrollo y del peso fetal por evaluación ecográfica, permite mediante la biometría fetal detectar oportunamente patrones de crecimiento fetal normal o anormal, permitiendo establecer medidas de prevención y vigilancia que se traducirán en un mejor pronóstico para la madre y el feto.

El establecer valores referenciales permitirá la creación de la curva normal del índice de pulsatilidad de la arteria uterina, que orientará a verificar que el flujo doppler se encuentra dentro de los parámetros normales, asegurándonos de este modo que el feto se encuentre en buenas condiciones, permitiendo entonces detectar fetos en riesgo según se encuentren por debajo del percentil 5 (macrosomía fetal) o por encima del percentil 95 (RCIU), para así prevenir y/o disminuir el riesgo de resultados adversos como las múltiples complicaciones maternas,

La ecografía doppler de las arterias uterinas ha sido ampliamente adoptada en la práctica clínica y es sin duda la prueba clínica más estudiada que se ha convertido en un método útil de evaluación indirecta de la circulación útero placentaria, considerando que resultados anormales se encuentran fuertemente relacionados con resultados maternos perinatales adversos.

El uso clínico del doppler de la arteria uterina necesita de la existencia de rangos referenciales normales para nuestra población. Índices de pulsatilidad promedio medidas entre las 11 a 41 semanas de embarazo que hasta el momento no se encuentran reportadas.

En el instituto materno perinatal no hay trabajos sobre valores referenciales en fetos sanos, por tal motivo se ve la necesidad de establecer los valores referenciales normales, para disminuir la morbimortalidad fetal $y$ neonatal. Además por ser una institución de referencia los datos obtenidos nos sirven como parámetro referencial a nivel nacional.

El presente estudio establece rangos referenciales para el percentil 5, 50 y 95 del índice de pulsatilidad de la arteria uterina. Demostrando que los IP entre los valores del percentil 5 y 95 tienen un valor clínico en la detección de fetos en buenas condiciones.

\section{MATERIALES Y MÉTODOS}

El estudio cuantitativo, observacional, descriptivo, transversal y retrospectivo se realizó en fetos sanos de madres de bajo riesgo a las que se realizó la medición del IP de la arteria uterina entre las 11 a 41 semanas en la Unidad de Medicina Fetal del Instituto Nacional Materno
Perinatal en el periodo comprendido en los años 2010 al 2016.

La población elegible fueron fetos sanos de madres de bajo riesgo con gestación única y edad gestacional entre 11 y 41 semanas, que tuvieron un recién nacido sano al término de su embarazo en el Instituto Nacional Materno Perinatal. Se excluyeron a los fetos con malformaciones fetales, restricción del crecimiento intrauterino y macrosomía fetal.

Los datos obtenidos por técnica de revisión de documentos de las historias clínicas completas y que cumplieron con los criterios de inclusión y exclusión, se registraron en una ficha elaborada para el presente estudio que fue validada por tres expertos en Gineco-obstetricia.

La recolección de datos se realizó previa autorización del Jefe de departamento de Medicina Fetal y del Servicio de Obstetricia del Instituto Nacional Materno Perinatal se procedió a la búsqueda de historias clínicas de pacientes a las que se les realizó un estudio Doppler donde se determinó el índice de pulsatilidad de la arteria uterina entre las 11 y 41 semanas de gestación.

Los datos obtenidos de las historias clínicas que cumplían con los criterios de inclusión se ingresaron a una base de datos creada en Excel. Para realizar la unificación de base de datos de la investigación con la base de datos de nacimientos del departamento de pediatría, se exportarón al programa de Acces, donde se seleccionó a las gestante que obtuvieron un recién nacido sano, la cual fue la población total de este estudio.

Los datos obtenidos durante la investigación por medio de la ficha de recolección de datos, se ordenarón y procesarón utilizando el programa de Excel. Los datos se ordenaron y se clasificaron de acuerdo a los objetivos e indicadores.

Para el análisis estadístico de los datos se utilizó el programa Excel. Para la variable cuantitativa (edad materna) se estimarón las medidas de tendencia central (Media, moda y mediana). La variable edad gestacional fue analizada como una variable cualitativa, donde se realizarón cuadros de distribución de frecuencia y porcentajes.

Los índices Doppler se analizarón en el programa SPSS por medio de transformación natural logarítmica de los valores del índice de pulsatilidad, seguida de la regresión por estimación curvilínea modelo inverso, pronosticando valores con un nivel de confianza del 95\%. Para lograr homogenizar los valores del IP a una misma edad gestacional, se realiza formula anti logarítmica, obteniendo valores representativos del IP. Los valores del P5, 50 y 95 de estos índices fueron calculados para cada edad gestacional entre las 11 a 41 semanas de gestación. Para la elaboración de los cuadros y gráficos se utilizó el programa Excel para Windows 2013. 


\section{RESULTADOS}

El estudio fue realizado con información recopilada entre los años 2010 a 2016, comprendiendo a 7020 gestantes que cumplieron con los criterios de inclusión y exclusión. El porcentaje de gestantes que se presentan con mayor frecuencia están entre las 13 y 14 semanas con un $9.07 \%$ y $9.10 \%$ respectivamente (Tabla 1 ).

Dentro de los resultados obtenidos en este estudio sobre el análisis según grupo de edad se encontró que el $47 \%$ (3314) del total de pacientes en el estudio presentaron edades entre 24 a 33 años y el $27 \%$ (1882) entre 34 a 43 años. Se presentó una moda de 27 , media de 29 y mediana de 29 (Tabla 2).

La Tabla 3 presenta los índices de pulsatilidad de la arteria uterina referenciales para cada edad gestacional en nuestra población. Se muestran los percentiles estimados $5^{\circ}, 50^{\circ}$ y $95^{\circ}$. Se observa una disminución progresiva del IP desde la semana 11 a la semana 41 . En el P5 el IP a las 11 semanas es de 1,13 , va disminuyendo progresivamente hasta llegar

Tabla 1. Análisis de frecuencia de las pacientes estudiadas instituto nacional materno perinatal 2010 a 2016 . Lima Perú.

\begin{tabular}{|c|c|c|}
\hline \multirow{2}{*}{ EG } & \multicolumn{2}{|c|}{ TOTAL } \\
\hline & $\mathbf{N}$ & $\%$ \\
\hline 11 & 151 & $2.15 \%$ \\
\hline 12 & 293 & $4.17 \%$ \\
\hline 13 & 637 & $9.07 \%$ \\
\hline 14 & 639 & $9.10 \%$ \\
\hline 15 & 397 & $5.66 \%$ \\
\hline 16 & 232 & $3.30 \%$ \\
\hline 17 & 160 & $2.28 \%$ \\
\hline 18 & 159 & $2.26 \%$ \\
\hline 19 & 281 & $4.00 \%$ \\
\hline 20 & 408 & $5.81 \%$ \\
\hline 21 & 207 & $2.95 \%$ \\
\hline 22 & 313 & $4.46 \%$ \\
\hline 23 & 335 & $4.77 \%$ \\
\hline 24 & 337 & $4.80 \%$ \\
\hline 25 & 194 & $2.76 \%$ \\
\hline 26 & 197 & $2.81 \%$ \\
\hline 27 & 136 & $1.94 \%$ \\
\hline 28 & 129 & $1.84 \%$ \\
\hline 29 & 81 & $1.15 \%$ \\
\hline 30 & 138 & $1.97 \%$ \\
\hline 31 & 135 & $1.92 \%$ \\
\hline 32 & 164 & $2.34 \%$ \\
\hline 33 & 172 & $2.45 \%$ \\
\hline 34 & 177 & $2.52 \%$ \\
\hline 35 & 205 & $2.92 \%$ \\
\hline 36 & 179 & $2.55 \%$ \\
\hline 37 & 191 & $2.72 \%$ \\
\hline 38 & 168 & $2.39 \%$ \\
\hline 39 & 120 & $1.71 \%$ \\
\hline 40 & 61 & $0.87 \%$ \\
\hline 41 & 24 & $0.34 \%$ \\
\hline TOTAL & 7020 & $100 \%$ \\
\hline
\end{tabular}

a la semana 32 en donde se presenta una disminución intercalada de 0 a 0.01 hasta llegar a la semana 41 con un IP de 0.41. En el P50 el IP a las 11 semanas es de 1.83, va disminuyendo progresivamente hasta llegar a la semana 35 con un IP de 0,70 (No se presentó disminución del IP entre las semanas 35 a 36 y la semana 39 a 40), culminando a las 41 semanas con un IP de 0,66. En el P95 el IP a las 11 semanas es de 2,96 va disminuyendo progresivamente hasta llegar a la semana 41 con un IP de 1,07.

Tabla 2. Análisis según grupo de edad instituto nacional materno perinatal 2010 a 2016. Lima - Perú

\begin{tabular}{ccc}
\hline GRUPOS DE EDAD & $\mathbf{N}$ & $\%$ \\
\hline$<14$ & 10 & $0.1 \%$ \\
$14-23$ & 1758 & $25 \%$ \\
$24-33$ & 3314 & $47 \%$ \\
$34-43$ & 1882 & $27 \%$ \\
$>43$ & 56 & $0.8 \%$ \\
TOTAL & 7020 & $100 \%$ \\
\hline
\end{tabular}

Tabla 3. Rangos de referencia para el índice de pulsatilidad de la arteria uterina en fetos sanos instituto nacional materno perinatal 2010 a 2016 . Lima - Perú.

\begin{tabular}{|c|c|c|c|}
\hline EG & P5 & P50 & P95 \\
\hline 11 & 1.13 & 1.83 & 2.96 \\
\hline 12 & 1.01 & 1.63 & 2.64 \\
\hline 13 & 0.92 & 1.48 & 2.39 \\
\hline 14 & 0.84 & 1.36 & 2.19 \\
\hline 15 & 0.78 & 1.26 & 2.04 \\
\hline 16 & 0.73 & 1.18 & 1.91 \\
\hline 17 & 0.69 & 1.12 & 1.81 \\
\hline 18 & 0.66 & 1.06 & 1.72 \\
\hline 19 & 0.63 & 1.02 & 1.64 \\
\hline 20 & 0.61 & 0.98 & 1.58 \\
\hline 21 & 0.58 & 0.94 & 1.52 \\
\hline 22 & 0.56 & 0.91 & 1.47 \\
\hline 23 & 0.55 & 0.88 & 1.43 \\
\hline 24 & 0.53 & 0.86 & 1.39 \\
\hline 25 & 0.52 & 0.84 & 1.35 \\
\hline 26 & 0.51 & 0.82 & 1.32 \\
\hline 27 & 0.50 & 0.80 & 1.29 \\
\hline 28 & 0.49 & 0.79 & 1.27 \\
\hline 29 & 0.48 & 0.77 & 1.24 \\
\hline 30 & 0.47 & 0.76 & 1.22 \\
\hline 31 & 0.46 & 0.74 & 1.20 \\
\hline 32 & 0.45 & 0.73 & 1.18 \\
\hline 33 & 0.45 & 0.72 & 1.17 \\
\hline 34 & 0.44 & 0.71 & 1.15 \\
\hline 35 & 0.44 & 0.70 & 1.14 \\
\hline 36 & 0.43 & 0.70 & 1.12 \\
\hline 37 & 0.43 & 0.69 & 1.11 \\
\hline 38 & 0.42 & 0.68 & 1.10 \\
\hline 39 & 0.42 & 0.67 & 1.09 \\
\hline 40 & 0.41 & 0.67 & 1.08 \\
\hline 41 & 0.41 & 0.66 & 1.07 \\
\hline
\end{tabular}

El gráfico 1 muestra el diagrama de estimación curvilínea del índice de pulsatilidad promedio de la arteria uterina por edad gestacional, representando los percentiles estimados $5^{\circ}, 50^{\circ}$ y $95^{\circ}$ en nuestra población. La media 
del índice de pulsatilidad de la arteria uterina muestra una disminución progresiva desde las 11 a 41 semanas.

\section{DISCUSIÓN}

Para la discusión se consideró como referencia algunos parámetros de trabajos realizadas en condiciones similares. El estudio evaluó la distribución de las mediciones del índice de pulsatilidad por cada edad gestacional en nuestra población.

El presente estudio muestra una disminución progresiva del IP desde la semana 11 a la semana 41 en los percentiles 5,50 y 95, así como en los estudios presentados por Gómez-Figueras y Borges Peixoto a diferencia del estudio presentado por Krampl en donde a partir de la semana 33 en su P50 el IP asciende llegando a la semana 40 con un IP de 0,79 (Tabla 4).

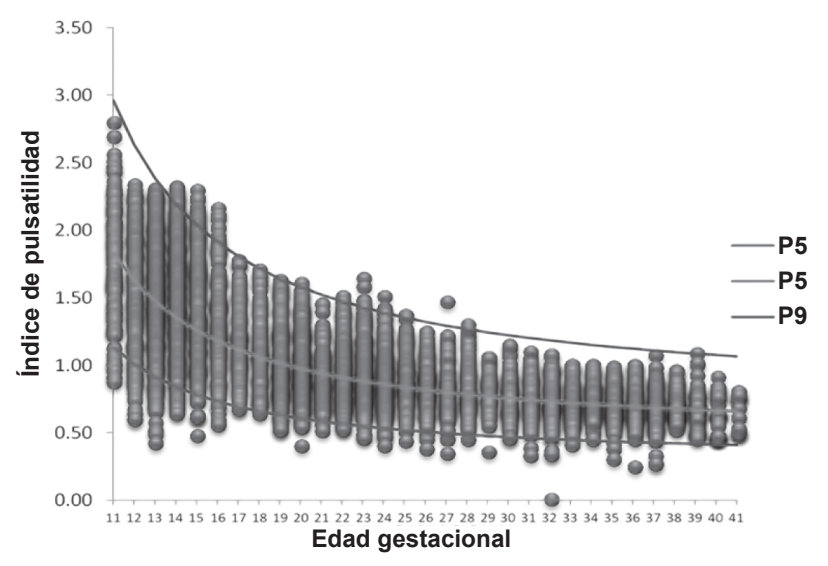

Gráfico 1. Diagrama de dispersión del índice de pulsatilidad promedio de las arterias uterinas según edad gestacional en fetos sanos del Instituto Nacional Materno Perinatal 2010 a 2016. Lima - Perú. Se presentan las curvas normales que representan el percentil 5, 50 y 95 .

Tabla 4. Rangos de referencia del índice de pulsatilidad de la arteria uterina según varios autores.

\begin{tabular}{|c|c|c|c|c|c|c|c|}
\hline EG & P5 INMP & $\begin{array}{c}\text { P5 Gómez- } \\
\text { Figueras }\end{array}$ & P50 INMP & $\begin{array}{l}\text { P50 Gómez- } \\
\text { Figueras }\end{array}$ & P50 Krampl & P95 INMP & $\begin{array}{l}\text { P95 Gómez- } \\
\text { Figueras }\end{array}$ \\
\hline 11 & 1.13 & 1.18 & 1.83 & 1.79 & - & 2.96 & 2.70 \\
\hline 12 & 1.01 & 1.11 & 1.63 & 1.68 & - & 2.64 & 2.53 \\
\hline 13 & 0.92 & 1.05 & 1.48 & 1.58 & - & 2.39 & 2.38 \\
\hline 14 & 0.84 & 0.99 & 1.36 & 1.49 & 1.71 & 2.19 & 2.24 \\
\hline 15 & 0.78 & 0.94 & 1.26 & 1.41 & 1.56 & 2.04 & 2.11 \\
\hline 16 & 0.73 & 0.89 & 1.18 & 1.33 & 1.44 & 1.91 & 1.99 \\
\hline 17 & 0.69 & 0.85 & 1.12 & 1.27 & 1.33 & 1.81 & 1.88 \\
\hline 18 & 0.66 & 0.81 & 1.06 & 1.20 & 1.23 & 1.72 & 1.79 \\
\hline 19 & 0.63 & 0.78 & 1.02 & 1.15 & 1.15 & 1.64 & 1.70 \\
\hline 20 & 0.61 & 0.74 & 0.98 & 1.10 & 1.08 & 1.58 & 1.61 \\
\hline 21 & 0.58 & 0.71 & 0.94 & 1.05 & 1.01 & 1.52 & 1.54 \\
\hline 22 & 0.56 & 0.69 & 0.91 & 1.00 & 0.96 & 1.47 & 1.47 \\
\hline 23 & 0.55 & 0.66 & 0.88 & 0.96 & 0.91 & 1.43 & 1.41 \\
\hline 24 & 0.53 & 0.64 & 0.86 & 0.93 & 0.86 & 1.39 & 1.35 \\
\hline 25 & 0.52 & 0.62 & 0.84 & 0.89 & 0.83 & 1.35 & 1.30 \\
\hline 26 & 0.51 & 0.60 & 0.82 & 0.86 & 0.80 & 1.32 & 1.25 \\
\hline 27 & 0.50 & 0.58 & 0.80 & 0.84 & 0.77 & 1.29 & 1.21 \\
\hline 28 & 0.49 & 0.56 & 0.79 & 0.81 & 0.75 & 1.27 & 1.17 \\
\hline 29 & 0.48 & 0.55 & 0.77 & 0.79 & 0.73 & 1.24 & 1.13 \\
\hline 30 & 0.47 & 0.54 & 0.76 & 0.77 & 0.72 & 1.22 & 1.10 \\
\hline 31 & 0.46 & 0.52 & 0.74 & 0.75 & 0.71 & 1.20 & 1.06 \\
\hline 32 & 0.45 & 0.51 & 0.73 & 0.73 & 0.70 & 1.18 & 1.04 \\
\hline 33 & 0.45 & 0.50 & 0.72 & 0.71 & 0.70 & 1.17 & 1.01 \\
\hline 34 & 0.44 & 0.50 & 0.71 & 0.70 & 0.71 & 1.15 & 0.99 \\
\hline 35 & 0.44 & 0.49 & 0.70 & 0.69 & 0.71 & 1.14 & 0.97 \\
\hline 36 & 0.43 & 0.48 & 0.70 & 0.68 & 0.72 & 1.12 & 0.95 \\
\hline 37 & 0.43 & 0.48 & 0.69 & 0.67 & 0.73 & 1.11 & 0.94 \\
\hline 38 & 0.42 & 0.47 & 0.68 & 0.66 & 0.75 & 1.10 & 0.92 \\
\hline 39 & 0.42 & 0.47 & 0.67 & 0.65 & 0.77 & 1.09 & 0.91 \\
\hline 40 & 0.41 & 0.47 & 0.67 & 0.65 & 0.79 & 1.08 & 0.90 \\
\hline 41 & 0.41 & 0.47 & 0.66 & 0.65 & - & 1.07 & 0.89 \\
\hline
\end{tabular}


En el P5 el IP a las 11 semanas es de 1,13, va disminuyendo progresivamente hasta llegar a la semana 32 (IP 0,45) en donde se presenta una disminución intercalada de 0 a 0,01 hasta llegar a la semana 41 con un IP de 0,41. En el estudio de Gómez-Figueras el IP a las 11 semanas es de 1.18, disminuye progresivamente hasta la semana 33 (IP 0,50) presentando disminución intercalada hasta la semana 38 donde se estaciona con un IP de 0,47 , que es el IP con el que llega a la semana 41. Estos valores señalan que el $\mathrm{P} 5$ del estudio presenta valores más bajos con respecto al P5 de Gómez-Figueras desde el inicio hasta el final del embarazo (Gráfico 2).

En el P50 el IP a las 11 semanas es de 1,83, disminuye progresivamente, culminando a las 41 semanas con un IP de 0.66. El estudio de Gómez-Figueras presenta un IP de 1,79 a las 11 semanas. Desde la semana 13 (IP 1,58) se presenta una diferencia significativa con el P50 de este estudio (IP 1,48). En la semana 32, ambos estudios presentan un IP de 0,73 , luego disminuyen, culminando a las 41 semanas el estudio de Gómez-Figueras con un IP de 0,65. El estudio de Krampl inicia con un IP de 1,71 a las 14 semanas (INMP 2010-2016: 14 semanas IP de 1,36), llega a una disminución máxima a las 32 semanas con un IP de 0.70 (INMP 2010-2016: 32 semanas IP de 0,73), para luego incrementarse llegando a las 40 semanas con un IP de 0.79 (INMP 2010-2016: 40 semanas IP de 0,67). Por tanto el presente estudio presenta valores bajos en relación al estudio Krampl. En relación al estudio de Gómez-Figueras presenta valores más bajos al inicio de la gestación con interacción desde la semana 32 hasta el final de la gestación (Gráfico 3).

En el P95 el IP a las 11 semanas es de 2.96 disminuye progresivamente hasta la semana 41 con un IP de 1,07. El estudio de Gómez-Figueras presenta un IP de 2,70 a las 11 semanas, valor más bajo que el presentado en este estudio. A las 13 semanas se produce un cruce de las curvas, con un IP de 2,39 en este estudio y un IP de 2,38 en el estudio de Gómez-Figueras, cuyo IP incrementa

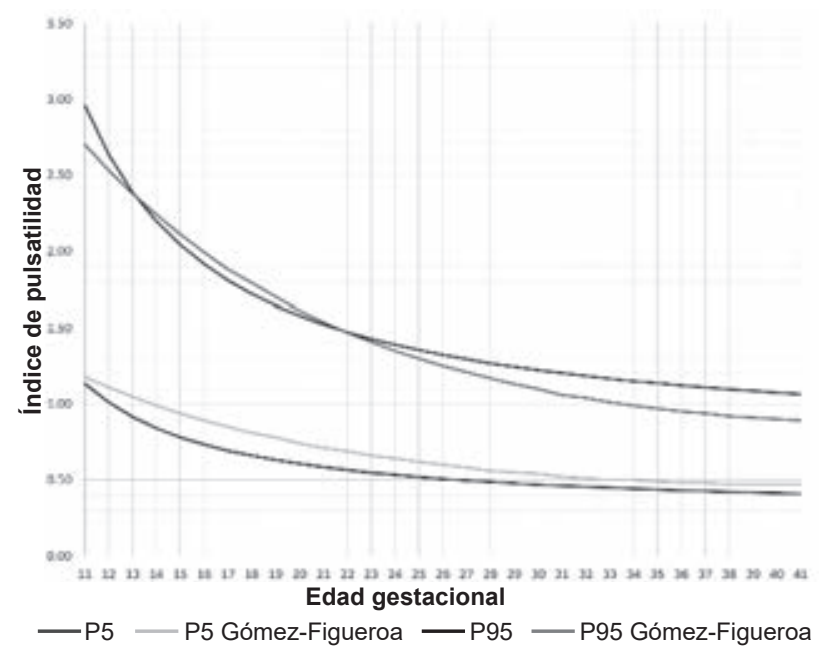

Gráfico 2. Curvas del percentil 5 y 95 del IP de la arteria uterina del INMP vs Gómez - Figueras.

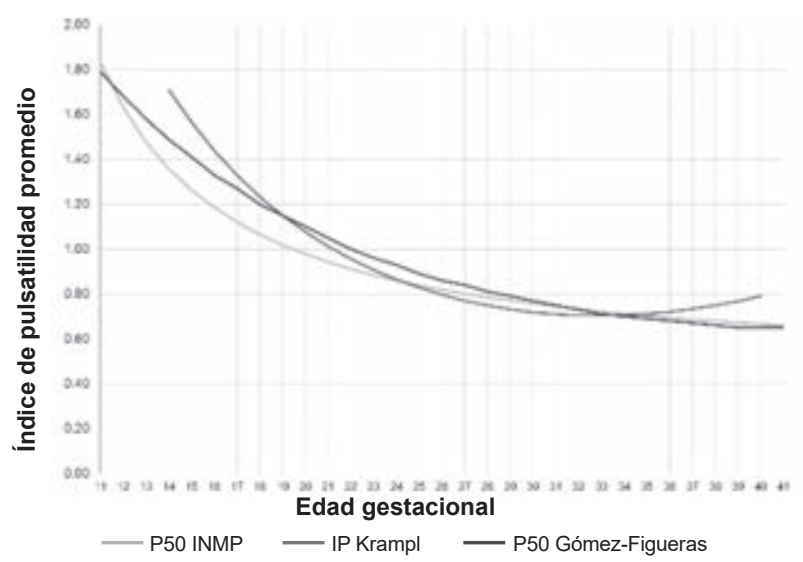

Grafico 3. Curvas del percentil 50 del IP de la arteria uterina del INMP vs Gómez - Figueras vs Krampl.

ligeramente hasta las 22 semanas donde se vuelven a cruzar ambas curvas con un IP de 1,47. Posteriormente ambas curvas disminuyen progresivamente en forma independiente, culminando el estudio de Gómez-Figueras con un IP de 0.89 a las 41 semanas. Lo cual señala que el presente estudio presenta valores más altos para el P95 a partir de la semana 23 en relación al estudio presentado por Gómez-Figueras (Gráfico 2).

En conclusión, se presentan rangos de referencia para el percentil 5, 50 y 95 del índice de pulsatilidad de la arteria uterina. Los IP encontrados entre el P5 y el P95 demuestran tener un valor clínico en la detección de fetos en buenas condiciones. Las curvas doppler del índice de pulsatilidad de las arterias uterinas en fetos sanos, muestra una disminución progresiva hasta las últimas etapas del embarazo.

Se recomienda la utilización del Doppler como medida de evaluación rutinaria en el monitoreo de la gestante de bajo y alto riesgo por el valor clínico que representa en la práctica obstétrica diaria. Las tablas de percentil 5,50 y 95 pueden ser consideradas como parámetro referencial a nivel nacional en la evaluación doppler de gestantes de bajo riesgo.

Financiamiento: autofinanciado.

Conflicto de interés: los autores declaran no tener algún conflicto de interés.

\section{REFERENCIAS BIBLIOGRÁFICAS}

1. Duan J, Chabot-Lecoanet AC, Perdriolle-Galet E, Chistov C, Hossu G, Cherifi A, Morel O. Vascularización úteroplacentaria en embarazos normales con preeclampsia y restricción del crecimiento intrauterino: Cuantificación en el tercer trimestre mediante doppler 3D en comparación con la morfología vascular placentaria: Un estudio prospectivo controlado. [Utero placental vascularisation in normal and preeclamptic and intra-uterine growth restriction pregnancies: third trimester quantification using 3D power Doppler with comparison to placental vascular morphology (EVUPA): a prospective controlled study]. BMJ Open [en línea]. 2016. 
[fecha de acceso 22 de octubre de 2016]; N6 URL disponible en: http://bmjopen.bmj.com/content/6/3/e009909

2. Ticona RM. Retardo de crecimiento intrauterino en el Perú según curva nacional de crecimiento fetal. Incidencia y resultados perinatales. ET VITA [en línea]. 2007. [fecha de acceso 14 de octubre 2016]; № 2 http://www.bvs.ins.gob.pe/ insprint/cindoc\%20/informes_tecnicos/73.pdf

3. Velázquez E. Morbimortalidad del recién nacido macrosómico Hospital III Suárez Angamos 2014 [Tesis de sub-especialidad en neonatología]. Lima: Repositorio académico Universidad San Martin de Porres; 2015.

4. Ticona-Rendón M, Pacora-PortellP, Huanco-Apaza D, TiconaVildoso M. Retraso del crecimiento intrauterino en el Perú: factores estresantes y resultados perinatales en hospitales del Ministerio de Salud. Ginecol Obstet Mex 2014; 82:725736.

5. Castillo W. Identificación de restricción del crecimiento intrauterino tardío con doppler umbilical normal mediante algoritmo diagnóstico multiparámetro en el instituto nacional materno perinatal. Revista Peruana de Investigación Materno Perinatal 2013; 2(1):19-22.

6. Limay $\mathrm{O}$. Índice de pulsatilidad promedio del doppler de las arterias uterinas como predictor de macrosomía fetal. Años 2011-2012. Revista Peruana de Investigación Materno Perinatal 2013; 2(1):8-13.

7. Ticona M, Huanco D. Características del peso al nacer en el Perú. Tacna-Perú: CONCYTEC; 2012.

8. Bujold E, Roberge S., Lacasse Y., Bureau M., Audibert F., Marcoux S., Forest J. C., Giguere Y. Prevención de la preeclampsia y la restricción del crecimiento intrauterino con aspirina comenzó en el embarazo temprano: Un metaanálisis. Obstet Gynecol. 2010; 116 (2): 402-14.

9. Akolekar R, Zaragoza EY, Poon L, Pepes S, Nicolaides K. Factor de crecimiento placentario sérico a las 11 a 13,6 semanas de gestación en la predicción de preeclampsia. Ultrasonido Obstet Gynecol. 2008; 32 (6): 732-9.

10. Lynn S, Kane S, Brennecke S, Da Silva F. Análisis doppler de la arteria uterina en el primer trimestre en la predicción de complicaciones posterior al embarazo. [First-Trimester Uterine Artery Doppler Analysis in the Prediction of Later Pregnancy Complicaciones]. Hindawi publiishing corporation [en línea]. 2015 [fecha de acceso 10 de setiembre de 2016]; No 679730 URL disponible en: https://www.hindawi.com/ journals/dm/2015/679730/

11. Barrios E, Martínez D, Torres A, Fajardo S, Panduro J. Tablas de referencia de biometría fetal para la población del occidente de México. Ginecol Obstet Mex. 2013; 81: 310320.

12. Doppler de la arteria uterina en el tamizaje ecográfico de semana 11-13.6 [base de datos en línea]. Argentina: Portal de educación permanente en ginecología y obstetricia; 2014. [fecha de acceso 22 de junio de 2016]. URL disponible en http://www.ms.gba.gov.ar/sitios/tocoginecologia/2014/05/29/ doppler-de-arteria-uterina-en-el-tamizaje-ecografico-desemana-11-a-136/

13. Krampl ER, Espinoza-Dorado J, Lees CC, Moscoso G, Bland JM, Campbell S. Estudio doppler de las arterias uterinas a gran altura y a nivel del mar. [Maternal uterine artery doppler studies at high altitude and sea level]. Ultrasound in Obstetrics \& Gynecology. 2001; 18: 578-582.

14. Gómez O, Figueras F, Fernández S, Bennasar M, Martinez JM, Puerto B, Gratacós E. Rangos de referencia del índice de pulsatilidad promedio de la arteria uterina en las 11 a 41 semanas de gestación. [Reference ranges for uterine artey mean pulsatility index at 11-41 weeks of gestación]. Ultrasound in Obstetrics \& Gynecology. 2008; 32(2): 128-132.
15. Da Costa A, Spara P, Oliveira T, Ramos W. Índices de resistencia y pulsatilidad de las arterias uterinas en el primer y segundo trimestre del embarazo normal. [Índices de resistência e pulsatilidade das artérias uterinas no primeiro e segundo trimestres de gestações normas]. Radiologia Brasileira [en línea].2010. [fecha de acceso 3 setiembre de 2016]; No3 URL disponible en: http:// www.scielo.br/scielo.php?script=sci_arttext\&pid=S010039842010000300006\#back

16. Borges A, Rodrigues T, Tonni G, De Almeida P, D'amico L, Martins W, Araujo E. Rangos referenciales del índice de pulsatilidad de la arteria uterina utilizando ultrasonido transvaginal a las 20 a 24.6 semanas de gestación en una población de Brasil de bajo riesgo. [Reference range for uterine artery Doppler pulsatility index using transvaginal ultrasound at 20-24w6d of gestation in a low-risk Brazilian populación]. Turk Ger Gynecol Assoc. 2016; 17: 16-20.

17. Utilidad del doppler de las arterias uterinas en el cribado de preeclampsia y restricción de crecimiento fetal. [base de datos en línea] Chile: Ultrasonografía y obstetricia temas selectos journal; 2007. [fecha de acceso 10 de junio de 2016]. URL disponible en: https://es.scribd.com/doc/314019935/ Utilidad-Del-Doppler-en-Obstetricia

18. Fleischer AC, Manning FA, Jeanty P, Romero R. Ecografía en obstetricia y ginecología. $7^{a}$ ed. Venezuela: Amolca, 2015.

19. Gonzales C. Simposio, preeclampsia, un viejo problema aún no resuelto: Nuevos conceptos. Revista Peruana de Ginecología y Obstetricia [en línea] 2014. [fecha de acceso 22 de junio de 2016]; No.4 URL disponible en: http://www.scielo.org.pe/scielo. php?pid=S2304-51322014000400012\&script=sci_arttext.

20. Información general sobre la hipertensión en el mundo [en línea]. Suiza: Ediciones de la Organización Mundial de la Salud; 2013. [fecha de acceso19 de junio de 2016]. URL disponible en: http://www.google.com.pe/url?sa=t\&rct=j\&q=\&es$r c=s \&$ source $=$ web \& $c d=2 \&$ ved $=0$ ahUKEwjy $1 v D-o b v P A h U-$ HFR4KHZp9Aj8QFgggMAE\&url=http\%3A\%2F\%2Fwww. who.int $\% 2$ Firis $\% 2$ Fbitstream $\% 2$ F $10665 \% 2$ F $87679 \% 2$ F $1 \%$ 2FWHO_DCO_WHD_2013.2_spa.pdf\&usg=AFQjCNFejcFQzUMiprYxjRE5ou3ovblo0Q

21. Gratacós E, Figueras F, Hernández E, Puerto B. Doppler en medicina fetal: Técnicas y aplicación clínica. Madrid: Editorial Médica Panamericana; 2012.

22. Guía Clínica: Doppler en medicina fetal. Hospital Clínica de Barcelona. [Internet]. España: Instituto Clínico de Ginecología, Obstetricia y Neonatología. [Consulta el 23 de junio del 2016]. Disponible a: http://www.annualreviews.org/doi/abs/10.1146/ annurev-pathol-121808-102149?journalCode=pathmechdis

23. Martin AM, Bindra R, Curcio I, Cicero S, Nicolaides $\mathrm{KH}$. Screening for pre-eclampsia and fetal growth restriction by uterine artery Doppler at 11-14 weeks of gestation. Ultrasound Obstet Gynecol 2001; 18: 583-6.

24. Sáez N, Carvajal J. Tamizaje y prevención de pre-eclampsia guiado por Doppler de arterias uterinas: revisión sistemática de la literatura. Rev Chil Obstet Ginecol. 2012; 77 (3): 235-242.

25. Kong V. Índice de pulsatilidad de las arterias uterinas como predictor de preeclampsia [Tesis Doctoral] Lima: Repositorio Académico USMP, Universidad de San Martin de Porres; 2015.

26. Polani P. Chromosomal and other genetic influences on firth weight variation. Associated Scientific Publisher 1974; $127-$ 164.

27. Bajo Arenas M. Ultrasonografía Obstétrica. España: Marban; 2008.

28. La obesidad materna y el recién nacido macrosómico en el Instituto Especializado Materno Perinatal, Lima 2003-2005 [base de datos en línea]. Perú: Centro de documentación 
Universidad San Marcos; 2013. [fecha de acceso 2 de octubre de 2016]. URL disponible en:

http://bases.bireme.br/cgi-bin/wxislind.exe/iah/online/?/sisScript=iah/iah. $x$ is\&src=google\&base $=$ LILACS\&lang $=p \& n e x-$ tAction=Ink\&exprSearch=724549\&indexSearch=ID\#refine

29. Limay O. Ecografía morfológica general obstétrica y ginecológica. [diapositiva]. Perú: Editorial de la Universidad Científica del Sur; 2012. 11 diapositivas.

30. Castillo R. Macrosomía Fetal. Revista Mexicana de Ultrasonido en Medicina. 2013; 18: 3-8.
31. Callaway L, Prins J, Chang A, Mclintyre H. The prevalence and impact of overweight and obesity in an Australian population. MJA 2006; 184: 56-59.

32. Savona Ventura C, Gatt M. Short-term obstetrics outcomes in obese maltese women. Int J Diabetes Metab 2006; 14: 88-91.

\section{Correspondencia:}

Dirección: Av. Javier Prado Este 175, Piso 3. Of. 304 -San IsidroLima.

Teléfono: (51 1) 447-8056 Celular: (51 1) 984-840625

E-mail: antoniolimay@hotmail.com

\section{ORCID iDs}

Oscar Antonio Limav Ríos Jaime Ingar Pinedo

Erasmo Huertas Tacchino

Walter Castillo Urquiaga

Walter Ventura Laveriano ai) https://orcid org/0000-0001-6012-3705

(ii) https://orcid.org/0000-0001-6472-7899

(di) https://orcid.org/0000-0002-9851-8419

(ii) https://orcid.org/0000-0002-1054-7398

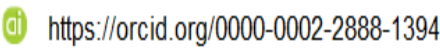

